\title{
Erfolgsbilanz: Brustzentren bieten hohe Behandlungsqualität
}

\author{
Etwa $90 \%$ aller Frauen, die an Brustkrebs erkranken, werden \\ inzwischen in zertifizierten Brustzentren behandelt. Die Therapie hat \\ sich dadurch verbessert - doch es gibt auch Kritik. Selbsthilfegruppen \\ bemängeln, dass Patientinnen oft zu früh entlassen werden.
}

n Deutschland gibt es 205 zertifizierte Brustzentren an 262 Standorten - das entspricht fast einer flächendeckenden Versorgung. Spätestens in zehn Jahren sollten alle Brustkrebs-Patientinnen in zertifizierten onkologischen Zentren behandelt werden. Das forderte der Präsident der Deutschen Krebsgesellschaft (DKG) Werner Hohenberger.

\section{Leitliniengerechte Tumortherapie in Brustzentren}

In zertifizierten Zentren erhielten Patienten eine leitliniengerechte, standardisierte Tumortherapie, betonte Rolf Kreienberg, Vorsitzender der Zertifizierungskommission Brustkrebszentren. Ein Beispiel: Bei drei von vier Patientinnen mit positivem Lymphknotenbefall wurde in den zertifizierten Zentren eine Chemotherapie eingesetzt. Die Leitlinien geben mindestens $60 \%$ vor. Dieser Sollwert werde mit 86,5\% der Brustzentren mehr als erfüllt, so Kreienberg.

Inzwischen erhielten $90 \%$ der Patientinnen ihre Primärtherapie in einem zertifizierten Brustzentrum. Doch die letzten $10 \%$ zu erreichen, sei schwierig, so Kreienberg: „Wir können niemanden zwingen, sich in einem Brustzentrum behandeln zu lassen. “ Nur mehr Aufklärung könne helfen, Patientinnen, aber auch die niedergelassenen Ärzte entsprechend von der Qualität zu überzeugen.

\subsection{Brustkrebs-Patientinnen befragt}

Aus Sicht der Experten ist die Behandlung in Zentren der richtige Weg: Schließlich erhalten die Brustkrebs-Patientinnen in den von der DKG und der Deutschen
Gesellschaft für Senologie (DGS) zertifizierten Zentren eine qualitativ hochwertige Versorgung. Das geht aus dem aktuellen Benchmarking-Bericht der beiden Gesellschaften hervor.

Befragt wurden 50.000 BrustkrebsPatientinnen an 246 Standorten in ganz Deutschland. Nach Angaben der Gesellschaften ist dies eine der größten Untersuchungen. Abgefragt wurden die leitliniengetreue Therapie sowie die interdisziplinäre Zusammenarbeit und die fachliche Expertise. Ein Maßstab hier: Die Zahl der Wundinfektionen.

\section{Frauen in Brustzentren gut aufgehoben}

„Die Ergebnisse der Studie spiegeln die hohe Qualität der Brustzentren wider“" betonte Rolf Kreienberg. So liege die Sollvorgabe bei Wundinfektionen in den Leitlinien bei unter $5 \%$. Die Brustzentren blieben jedoch mit einem mittleren Wert von $1,7 \%$ deutlich darunter, so Kreienberg. Anhand der Leitlinien seien zudem „Ausreißer nach oben“" schnell ausfindig zu machen. Diese Zentren würden aufgefordert, ihre Ergebnisse innerhalb einer Frist von drei Monaten bis zu einem Jahr - je nach Schwere des Fehlers - zu verbessern. Dadurch sei es „ein lernendes System“, sagte er.

Bei gravierenden Mängeln könne das Zentrum auch das Qualitätssiegel verlieren. Die Frauen scheinen sich in den Brustzentren jedenfalls gut aufgehoben zu fühlen: $95 \%$ der Betroffenen hatten das Gefühl, sich auf ihren Arzt verlassen zu können. Das ergab eine von der DKG in Auftrag gegebene Befragung mit mehr als 7.000 Teilnehmerinnen.

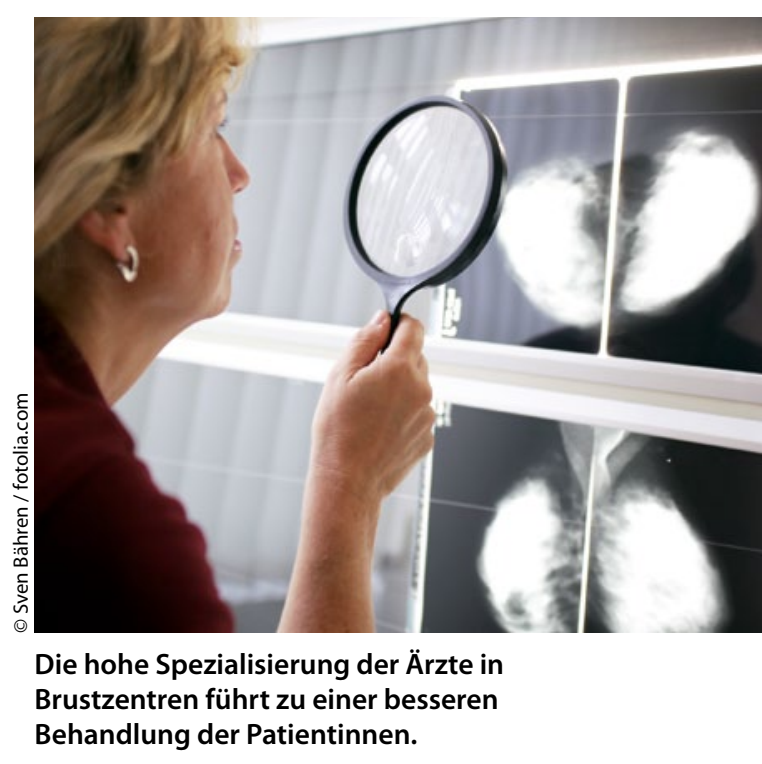

Zu frühe Entlassung kritisiert

Doch nicht alle Ergebnisse sehen so rosig aus: „Es gibt zum Beispiel deutliche Unterschiede zwischen den einzelnen Zentren“, sagte Holger Pfaff vom Institut für Medizinsoziologie der Universität Köln. Vor allem bei der Entlassung und der weiteren Behandlung beim niedergelassenen Arzt gebe es bei einigen Zentren noch Verbesserungspotenzial.

Die stellvertretende Bundesvorsitzende der „Frauenselbsthilfe nach Krebs“, Karin Meißler, kritisierte, dass immer noch viele Frauen am ersten Tag nach einer brusterhaltenden Operation entlassen würden: „Und zwar aus ökonomischen Gründen.“ Da sei das „Maß des Guten bei Weitem überschritten“, sagte Meißler. Durch eine so frühe Entlassung kämen die Patientinnen kaum dazu, Fragen zu stellen, auch fehle die Zeit für eine psychosoziale Betreuung.

Sunna Gieseke

Bericht von der Pressekonferenz ${ }_{\text {IV }}$ Versorgungsqualität an zertifizierten Brustkrebszentren" von der Deutschen Krebsgesellschaft und der Deutschen Gesellschaft für Senologie, Berlin, 2. Dezember 2011 